\title{
Divertor power deposition and target current asymmetries during type-I ELMs in ASDEX Upgrade and JET
}

\author{
$\underline{\text { T. Eich }}^{\mathrm{a}}$, A. Kallenbach ${ }^{\mathrm{a}}$, R.A. Pitts ${ }^{\mathrm{b}}$, S. Jachmich ${ }^{\mathrm{c}}$, J.C. Fuchs ${ }^{\mathrm{a}}$, A.Herrmann ${ }^{\mathrm{a}}$, J.Neuhauser ${ }^{\mathrm{a}}$ \\ ASDEX Upgrade Team and JET-EFDA contributors ${ }^{+}$ \\ a Max-Planck Institut fuer Plasmaphysik, Euratom Association, Garching, Germany \\ b Centre de Recherches en Physique des Plasmas, Association EURATOM, Conférédation Suisse, EPFL, 1015 \\ Lausanne, Switzerland \\ c Laboratory for Plasmaphysics, Ecole Royale Militaire/Koninklijke Militaire School, EURATOM-Association \\ "Belgian State", Brussels, Belgium, Partner in the Trilateral Euregio Cluster (TEC)
}

\begin{abstract}
Analysis of the type-I ELM power load asymmetries using infra-red thermography and target current measurements is performed ASDEX Upgrade Upper Single Null and JET DOC-L type-I ELMy H-Mode discharges with 'normal' and 'reversed' field direction, i.e. with the ion $B \times \nabla B$ drift direction pointing towards the active $\mathrm{X}$-point and the ion $B \times \nabla B$ drift direction pointing away from the active X-point, respectively. The ELM power load towards the inner target plate is found to be larger as towards the outer target with 'normal' field direction and vice versa with 'reversed' field. Current measurements are performed in ASDEX Upgrade providing information that a net negative charge flows into the outer target and a net positive charge into the inner target during the ELM in 'normal' field and vice versa for discharges with 'reversed' field. The difference between the ELM energy load on the inner and outer target, $E_{\text {outer }}-E_{\text {inner }}$, is well correlated with the measured charge flowing through the targets due to the ELM. A comparison to JET data shows that in both devices the maximum asymmetry in energy load corresponds to values of $E_{\text {inner }} / E_{\text {outer }} \approx 2$.
\end{abstract}

\footnotetext{
+ see appendix of J. Pamela, Fusion Energy 2004 (Proc. 20th Int. Conf. Vilamoura, 2004) IAEA, Vienna (2004).
} 
PACS: 52.55 Fa; 52.25.Vy; 52.55.Rk;52.40.Hf

JNM keywords: ELMs, Power deposition, target currents

PSI-16 keywords: ELMs, Power deposition, target currents, JET, ASDEX Upgrade

*Corresponding author address: Max-Planck-Institut für Plasmaphysik, Boltzmannstr.2, 85748, Garching, Germany

*Corresponding author E-mail: Thomas.eich@ipp.mpg.de

Presenting author: Thomas Eich

Presenting author e-mail: $\quad$ Thomas.eich@ipp.mpg.de 


\section{Introduction and experimental data base}

The target power deposition during edge localized modes [1] (ELMs) is a concern for the divertor target plates [2,3] in ITER. For an extrapolation of target power load characteristics (measured by infra-red thermography) of present devices such as ASDEX Upgrade and JET to ITER it is necessary to understand the ELM related SOL transport physics. For this reason, in JET and ASDEX Upgrade dedicated discharges for optimized infra-red measurements have been performed, complemented by measurements of currents flowing from the target plates to earth for both inner and outer divertor separately in ASDEX Upgrade [4]. Current losses due to ELMs have been reported and discussed for various devices [5-8,21]. To study the effect of ELM loss size and particle drifts on type-I ELM SOL transport, experiments were performed with varying heating power, plasma density and field direction. The field direction with the ion $B \times \nabla B$ drift direction pointing towards the active $\mathrm{X}$-point will be named as 'normal' throughout the paper, and the ion $B \times \nabla B$ drift direction pointing away from the $\mathrm{X}$-point as 'reversed'. It should be noted that the field direction change in ASDEX Upgrade is achieved by switching only the toroidal magnetic field whereas in JET both the direction of the toroidal magnetic field and the toroidal plasma current direction are switched. However, from the given data set in ASDEX Upgrade and in JET no distinction between the influence of drifts and the influence of field line orientation in relation to the plasma current direction can be made. The field line intersection angle on the target tile surfaces does not play a role since for JET the helicity is not changed and for ASDEX Upgrade the divertor target tiles are not tilted in toroidal direction.

Although progress was made for quantifying the ELM target load characteristics as expected for ITER $[9,18]$, the underlying transport mechanism driving a larger fraction of the ELM released energy towards the inner target plates than to the outer in 'normal' field direction is not resolved. This paper focuses on the latter issue and presents a correlation of the ELM target deposited energy asymmetries with the net target charge flowing through the inner and outer targets due to ELMs.

Co-deposited surface layers can influence the correct estimation of power fluxes from surface temperature measurements [10]. These layers are reported from JET [11] to be deposited largely asymmetrically on the inner and outer target tiles depending on the field direction. These influences have been minimized in ASDEX Upgrade upper single null discharges by installing new and therefore clean target tiles (for details see [12]). All presented data are 


\section{PSI17-Paper-T.Eich}

obtained by using coherent averaging techniques, in which about 10-30 Type-I ELMs are used for one data point in this work. The reader should note that all reported ELMs, following the definition in [1], are Type-I.

\section{Power deposition and target currents during type-I ELMs}

Before the ELM deposited target energy and target current are presented, the corresponding value for the Inter-ELM transport should be briefly discussed for both field directions. In the Inter-ELM phases with 'normal' field, generally a thermo electric current is observed caused by the difference of the local electron temperature, $T_{e}$, between both target plates with $T_{e}$ being reported to be larger at the outer target $[4,13,14]$. Also commonly observed, a larger fraction of the Inter-ELM released power into the SOL is deposited on the outer divertor target plates due to toroidal geometry effects and an increased radial transport at the outer equatorial midplane region. This ballooning-like power release is reported, based on experiments in Double Null magnetic configuration, not to change during the ELM energy release [12]. In 'reversed' field cases, the Inter-ELM target power deposition is reported to be roughly equal at the inner and outer target [12]. Measured inter-ELM thermo currents change flow direction for 'reversed' field and are interpreted to be caused by a larger $T_{e}$ at the inner target for that case. It should be noted that no plasmas are included in the analysis for ASDEX Upgrade in which one or both of the divertor legs are strongly detached in the inter-ELM phases.

Figure 1 (a) shows the experimental set up for the power flux and target current measurements in ASDEX Upgrade upper single null discharges for 'reversed' field direction. Figure 1(b) shows that during the ELM a larger fraction of the ELM energy is deposited on the outer target than on the inner (inverse to the inter-ELM ratio) and simultaneously that the observed target current is increased but keeping the same flow direction as in the Inter-ELM phase. Figure 1(c) shows the time integrals of the values presented in (b) namely the ELM energy for inner, $E_{\text {inner }}$, and outer target, $E_{\text {outer }}$, and the ELM integrated charge, $C_{E L M}$, flowing into the target plates; Note that $C_{E L M}$ is positive in the latter case, i.e. a positive current flows from the inner to the outer target plate through the SOL during the ELM.

In a similar discharge in ASDEX Upgrade but with 'normal' field ELMs are observed to impose larger value for $E_{\text {inner }}$ than for $E_{\text {outer }}$ and simultaneously negative value for $C_{E L M}$. Since the poloidal origin of the energy release location has not significantly changed from the 
Inter-ELM to the ELM phase, it may surprise that a larger fraction of the ELM energy is deposited onto the inner divertor target for the case with 'normal' field.

Summarizing these findings a lower ELM energy load is observed on that target plate where a net negative charge integrated over the ELM duration flows into that target. A net positive charge integrated over the ELM duration of same size within the error bars is measured for the target with the higher ELM energy load. Consequently, the target with lower value for $T_{e}$ at the divertor target plates in the Inter-ELM phases receives the larger power load during the ELM.

\section{Correlation of ELM target energy with target charge}

Figure 2 shows the correlation between the difference of ELM target load for outer and inner target, $E_{\text {outer }}-E_{\text {inner }}$, and net charge $C_{E L M}$ flowing through the targets integrated over the ELM duration. As it is obvious from the plot, both quantities are well correlated. Different aspects of the correlation should be noted by the reader. First, both quantities strictly change sign with field direction. Secondly, the graph passes through zero for $E_{\text {outer }}-E_{\text {inner }}=0$, i.e. for a balanced ELM target power load no ELM related currents are measured (within the error bars). Finally, the gradient of the graph for ,normal' and ,reversed' field are different roughly by a factor or two. By focusing in the following analysis on the ,normal' field direction the ratio between energy and charge is revealed from the gradient of the graph in Figure 3 with $\frac{E_{\text {outer }}-E_{\text {inner }}}{C_{E L M}}=\frac{-5 k J}{-5 A s}$ Equation (1).

Thermo electric currents are observed in ASDEX Upgrade for 'normal' field direction to be caused by a larger $T_{e}$ at the outer target than at the inner $[4,15]$ in Inter-ELM phases and vice versa for 'reversed' field. Figure 1 shows that for 'reversed' field the target currents during ELMs only increase in amplitude but otherwise do not change the sign (see also [6,7]). The same observation is made for 'normal' field. Therefore purely thermo electric currents as explained for the Inter-ELM phases are not consistent with the observed asymmetry of ELM energy load between inner and outer target. Particle drifts are a likely origin of the observed asymmetries. The different values of the energy to charge ratio during ELMs for the different field directions shown in Figure 2 are believed to be caused by a different interaction of the toroidal magnetic field direction dependent poloidal drift terms and the non toroidal magnetic 
field dependent terms causing in/out asymmetries which are related to the toroidal geometry and the corresponding ballooning-like power release.

Unfortunately no probe measurements are available for the presented data base of upper single null discharges in ASDEX Upgrade, so no quantitative analysis with respect to power flows in the electron and ion channels can be performed. Probe measurements from JET [16] performed for 'normal' and 'reversed' field also report a change of the sign of the ELM integrated target currents consistent to the findings for ASDEX Upgrade.

Relating the energy to charge difference as stated in equation (1) to single particles a value of $500 \mathrm{eV}$ per charge is found. It should be noted in that respect that the pedestal temperature for the discharges from the data base is about $500-700 \mathrm{eV}$. Together this may lead to the conjecture that the energy asymmetry is related to an in-out asymmetry of deposited pedestal ions (see also [17] in that respect).

\section{Comparison of ASDEX Upgrade and JET results}

Figure 4 shows a comparison of ASDEX Upgrade and JET values of $E_{\text {outer }}+E_{\text {inner }}$ versus $E_{\text {outer }}-E_{\text {inner }}$ for ,normal' and ,reversed‘ field direction. For ASDEX Upgrade ELMs with target load energies from $2 \mathrm{~kJ}$ to $20 \mathrm{~kJ}$ (this number can be verified in Figure 4) it is observed that $-1 / 3 \times\left(E_{\text {outer }}+E_{\text {inner }}\right) \leq E_{\text {outer }}-E_{\text {inner }} \leq 0$ for ,normal` and $0 \leq E_{\text {outer }}-E_{\text {inner }} \leq 2 / 3 \times\left(E_{\text {outer }}+E_{\text {inner }}\right)$ for ,reversed' field discharges. Obviously there is an unidentified parameter varying the $E_{\text {outer }}-E_{\text {inner }}$ value for each given $E_{\text {outer }}+E_{\text {inner }}$ value. A comparison of $E_{\text {outer }}-E_{\text {inner }}$ values to the pre-ELM pedestal top values of $T_{e}$, Greenwald density fraction, pedestal collisionality and the normalized ELM pedestal loss size did not reveal a simple correlation.

For JET the ELM target load energies cover values between $40 \mathrm{~kJ}$ to $500 \mathrm{~kJ}$. For the ,reversed“ field conditions more ELM energy is found to be deposited on the outer target and for ,normal' field cases more on the inner target identical to the findings for ASDEX Upgrade. The data base for 'reversed' field is otherwise very poor and therefore not further discussed here. For 'normal' field direction and again identical to ASDEX Upgrade the relation $-1 / 3 \times\left(E_{\text {outer }}+E_{\text {inner }}\right) \leq E_{\text {outer }}-E_{\text {inner }} \leq 0$ is found. For ELMs with target energies above $100 \mathrm{~kJ}$ values only a comparable small variation of the $E_{\text {outer }}-E_{\text {inner }}$ values is observed and $E_{\text {outer }}-E_{\text {inner }} \approx-1 / 3 \times\left(E_{\text {outer }}+E_{\text {inner }}\right)$ is found. Note that the variation of the ELM target load 
data at JET with target energies below $100 \mathrm{~kJ}$ could also be caused by the larger relative diagnostic error bars in JET (which arise due to the weaker infra-red camera temperature resolution and possibly due to influences of surface layers [10,11]). However, it seems plausible to speculate in that respect, that the unidentified parameter plays a significant role for all ELMs in ASDEX $(<20 \mathrm{~kJ})$ and for low energy ELMs $(<100 \mathrm{~kJ})$ in JET but not for large ELMs $(>100 \mathrm{~kJ})$ in JET. The corresponding value for $E_{\text {inner }} / E_{\text {outer }}$ are calculated here for the largest ELM in JET for ,normal` field by

$E_{\text {outer }}-E_{\text {inner }} \approx-1 / 3 \times\left(E_{\text {outer }}+E_{\text {inner }}\right) \Rightarrow \frac{E_{\text {inner }}}{E_{\text {outer }}} \approx 2$ Equation (2).

Only speculations are possible about the nature of the unidentified parameters which affect largely the ELM energy asymmetry for ASDEX Upgrade but have no effect on the values for large ELMs at JET. A diagnostically introduced scatter for ASDEX Upgrade is definitely excluded. A possible hint is given by the level of radiation (which appears mainly in the divertor regions) during ELMs and the fact that the absolute size of the ELMs in JET (e.g. deposited target energy or pedestal loss energy) is about a factor of 10-20 larger than in ASDEX Upgrade. In ASDEX Upgrade the radiated ELM energy can be of the same order of the deposited ELM energy or as less as 20\% [19]. The same value for type-I ELMs in JET is reported to be never to exceed values of $10 \%$ [20]. Therefore, a main suspect for the observed difference between ASDEX Upgrade and JET is the role of the SOL and divertor parameters.

\section{Conclusions}

Several conclusions can be drawn from the presented observations. First, ELMs drive a larger fraction of energy to be deposited in 'normal' field direction towards the inner divertor target and a larger fraction of energy to be deposited in 'reversed' field direction towards the outer divertor target. Secondly, the ELM energy difference is correlated to the charge flowing into the divertor target plates during the ELM and cannot be explained by thermo electric currents as they are evident for Inter-ELM phases [13]. Thirdly, the energy to charge ratio as found for the two field directions with 'normal' and 'reversed' field shows different absolute numbers. Finally, one or more unidentified parameter appear particularly for all ( $E_{\text {outer }}+E_{\text {inner }}<20 \mathrm{~kJ}$ ) ELM data reported for ASDEX Upgrade which seem to play no role for very large $\left(E_{\text {outer }}+E_{\text {inner }}>100 \mathrm{~kJ}\right)$ ELMs in JET. 


\section{References:}

[1] H.Zohm, Plasma Phys. Control. Fus. 38 (1996) 105.

[2] D.J. Campbell et al., Phys. Plasmas 8 (2001) 2041.

[3] G. Federici et al., Plasma Phys. Control. Fus. 45 (2003) 1523.

[4] A.Kallenbach et al., Journal Nucl. Mater., 290-223 (2001) 639.

[5] J.Lingertat et al., Journal Nucl. Mater., 241-242 (1997) p. 402

[6] R.A. Pitts et al., Nucl. Fus. 43 (2003), p. 1145

[7] H.Takahashi et al. Nucl. Fusion 44 (2004), p.1075

[8] P.Stangeby, Nucl.Fusion 43 (2003), p.L1-L5

[9] T. Eich et al. J. Nucl. Mat. 337-339 (2005) 669.

[10] A.Herrmann, Plasma Phys. Control. Fus. 44 (2002) 883.

[11] P.Andrew et al., Journal Nucl.Mater., 313-316 (2003), p.135

[12] T.Eich et al., Plasma Phys. Control. Fus. 47 (2005) 815.

[13] P.J. Harbour, Contr.Plas.Phys. 28 (1988), p. 417

[14] G.M.Staebler and F.L.Hinton, Nucl.Fusion 29 (1989) p.1820

[15] U. Wenzel at al., Nucl. Fus. 41 (2001), p. 1695

[16] S.Jachmich et al., these proceedings

[17] W. Fundamenski, R.A. Pitts et al., Plasm.Phys. Contr. Fus. 48 (2006) 109.

[18] A.Loarte et al., Physics of Plasmas, 11 (2004), p. 2668

[19] J.C.Fuchs et al., Journal Nucl. Mater. 337-339 (2005), p.756-760

[20] P.Monier-Garbet, Nucl.Fusion 45 (2005), p.1404

[21] D.Testa et al., Plasma Phys. Control. Fus. 47 (2005) 733. 


\section{Figure captions}

Figure 1: (a) Sketch of inner/outer ELM power load asymmetry and corresponding target current direction for ASDEX Upgrade Upper Single Null discharges with 'reversed' ion $B \times \nabla B$ drift direction pointing away from the active (upper) $\mathrm{X}$-point. (b) Power load for inner (red) and outer (blue) target plates and target current evolution during type-I ELM in ASDEX for coherently averaged data of about 20 ELMs. (c) Time integrals of the values in (b) over ELM duration in same colours.

Figure 2: Correlation between the measured difference of ELM deposited energies towards inner and outer divertor target, $E_{\text {outer }}-E_{\text {inner }}$, with the ELM related charge difference $C_{E L M}$. Note that both values, $E_{\text {outer }}-E_{\text {inner }}$ and $C_{E L M}$ strictly change sign with field direction. Open symbols are 'reversed' field data and closed symbols 'normal field'.

Figure 3: Correlation of the ELM deposited energy for both targets, $E_{\text {outer }}+E_{\text {inner }}$ with $E_{\text {outer }}-E_{\text {inner }}$ in ASDEX Upgrade and JET. (b) In JET the data points for large ELMs $(>100 \mathrm{~kJ})$ in 'normal' field are close to $E_{\text {inner }} / E_{\text {outer }}=2$. Open symbols are 'reversed' field data and closed symbols 'normal field'. 


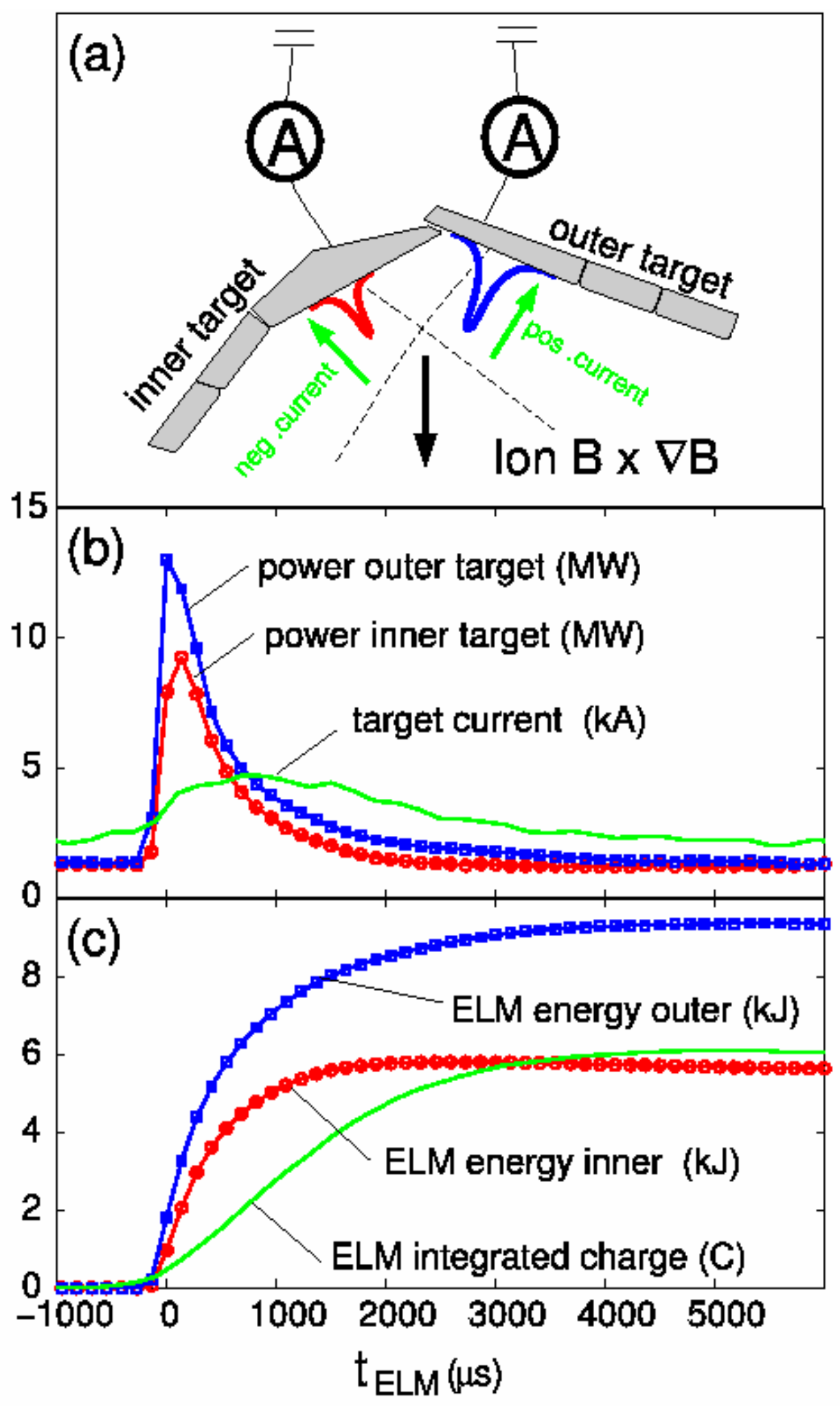

Figure 1

(75 mm wide figure) 


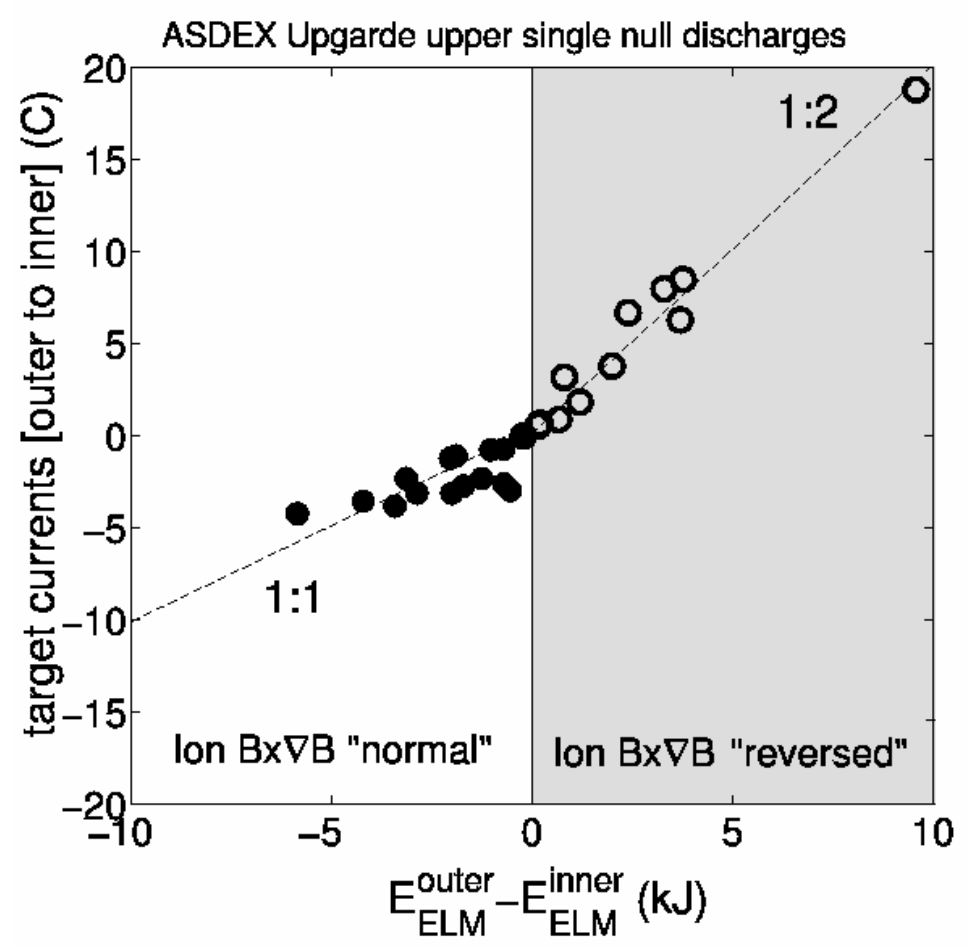

Figure 2

(75 mm wide figure)
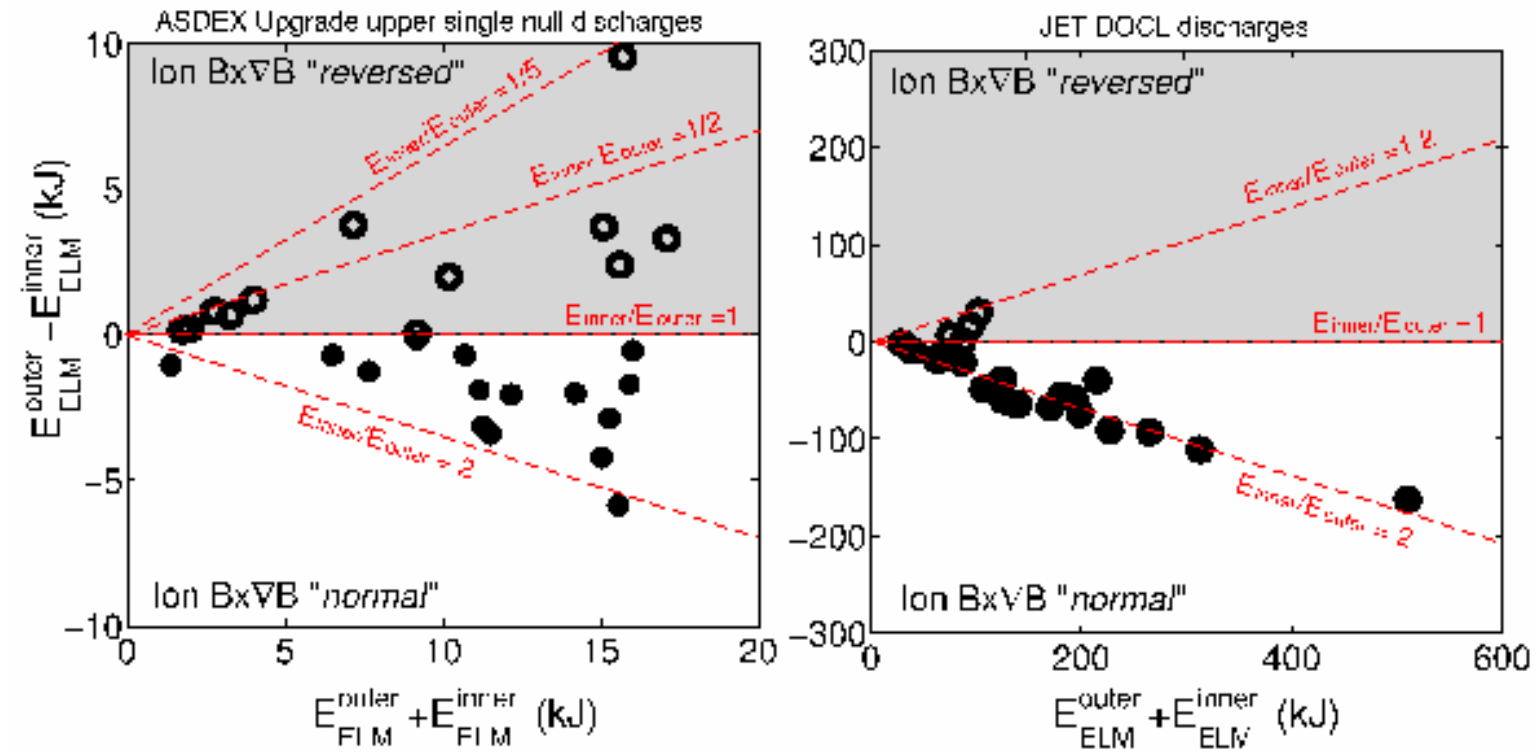

Figure 3 
PSI17-Paper-T.Eich

(160 mm wide figure) 